\title{
In Situ Laser Synthesis of 2D WSe2 Within TEM
}

Chenze Liu ${ }^{1}$, Yu-Chuan Lin ${ }^{2}$, Yiling Yu ${ }^{2}$, Alexander Puretzky ${ }^{2}$, Mina Yoon ${ }^{2}$, Gyula Eres ${ }^{2}$, Christopher Rouleau $^{2}$, Kai Xiao ${ }^{2}$, David Geohegan ${ }^{2}$ and Gerd Duscher ${ }^{1}$

${ }^{1}$ University of Tennessee-Knoxville, Knoxville, Tennessee, United States, ${ }^{2}$ Oak Ridge National Laboratory, Oak Ridge, Tennessee, United States

Non-equilibrium synthesis and processing enables a wide variety of materials systems with tuned metastable phases and properties. Here we report how a prototype setup allows laser illumination to be coupled into transmission electron microscopes (TEM) for real-time observations of two-dimensional (2D) materials synthesis and processing. The configuration and ability of in situ photon delivery system was reported previously ${ }^{1-2}$. Amorphous atoms and molecules were deposited at room temperature on TEM grids by pulsed laser deposition. They were subsequently irradiated by a laser built within a highresolution TEM (HRTEM) and then crystallized through various pathways observed by in situ TEM imaging. In addition, post-growth high-angle annular dark field (HAADF) Z-contrast scanning TEM (STEM) measurements are conducted for characterization of crystal phases, defects and grain boundaries at the atomic scale.

Two-dimensional WSe2 was synthesized from amorphous precursors within the TEM with laser irradiation. The final morphology and the growth pathways were found to be dependent on the substrate properties. Figure 1a shows the post-growth atomic resolution HAADF image of 2D WSe 2 grown on graphene by laser irradiation (wavelength $=785 \mathrm{~nm}$, pulse width $=10 \mathrm{msec}$ ). The final $2 \mathrm{D} \mathrm{WSe} 2$ crystals are polycrystalline, evident by the selected area electron diffraction (SAED) pattern and also the fast Fourier transformation (FFT) of an overview STEM image. Conversely, WSe2 crystals grown on chemical vapor deposition (CVD) MoSe2 monolayer substrate by laser present a perfect alignment with the MoSe2, confirmed by the SAED pattern and FFT image (Figure 1b). We attribute this improvement in van der Waals epitaxy to the reduced lattice mismatch from WSe2/graphene (25\%) to WSe2/MoSe2 (0.2\%). STEM images also show the twisted angles between $\mathrm{WSe}_{2}$ and $\mathrm{MoSe}_{2}$ heterobilayer are $0^{\circ}(3 \mathrm{R}), 60^{\circ}(2 \mathrm{H})$, or $\leq$ $5^{\mathrm{o}}$.

The crystallization pathway of 2D WSe2 by laser irradiation within the TEM is shown in Figure 2. On graphene (Figure 2a), prior to become the 2D planar structure, 3D metastable WSe2 was achieved in a Serich environment. Similar 3D metastable phase has been found during the formation of 2D MoS 2 flakes from the thermolysis of ammonium thiomolybdates on $\mathrm{Si}_{3} \mathrm{~N}_{4}$ membrane using in situ heating $\mathrm{TEM}^{3}$. The structural evolution was found to largely depend on Se to W ratio during laser irradiation. On $\mathrm{MoSe}_{2}$ (Figure 2b), prior to form epitaxial WSe2, crystallites nucleate with different orientations first and then register into the lattice of $\mathrm{MoSe}_{2}$. The temperatures of the substrate corresponding to different laser energies were measured by the shifts of their exciton peaks using electron energy loss spectroscopy (EELS) at cryogenic temperatures in a mono-chromated TEM. The minimum crystallization temperature for WSe2 was measured to be $300{ }^{\circ} \mathrm{C}$. 
Notice: This abstract has been authored by UT-Battelle, LLC, under Contract No. DE-AC05-00OR22725 with the U.S. Department of Energy. The United States Government retains and the publisher, by accepting the article for publication, acknowledges that the United States Government retains a nonexclusive, paid-up, irrevocable, world-wide license to publish or reproduce the published form of this manuscript, or allow others to do so, for United States Government purposes. The Department of Energy will provide public access to these results of federally sponsored research in accordance with the DOE Public Access Plan ( http://energy.gov/downloads/doe-public-access-plan ).
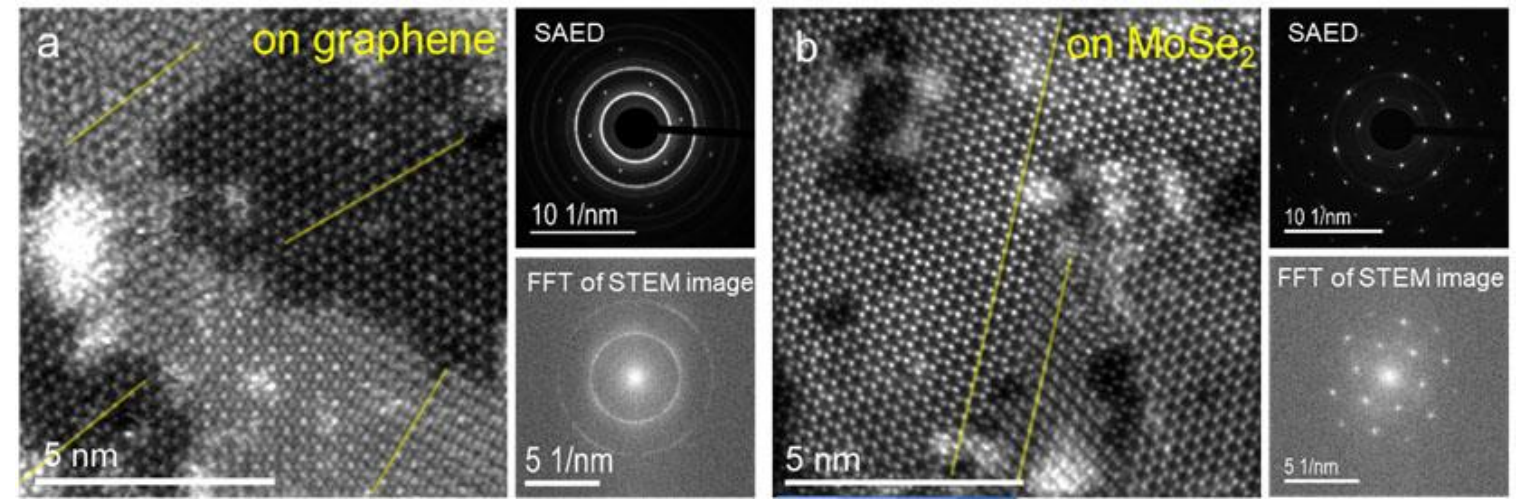

Figure 1. Post-growth morphology of 2D WSe2 grown by laser irradiation within the TEM on (a) graphene and (b) CVD grown monolayer MoSe2

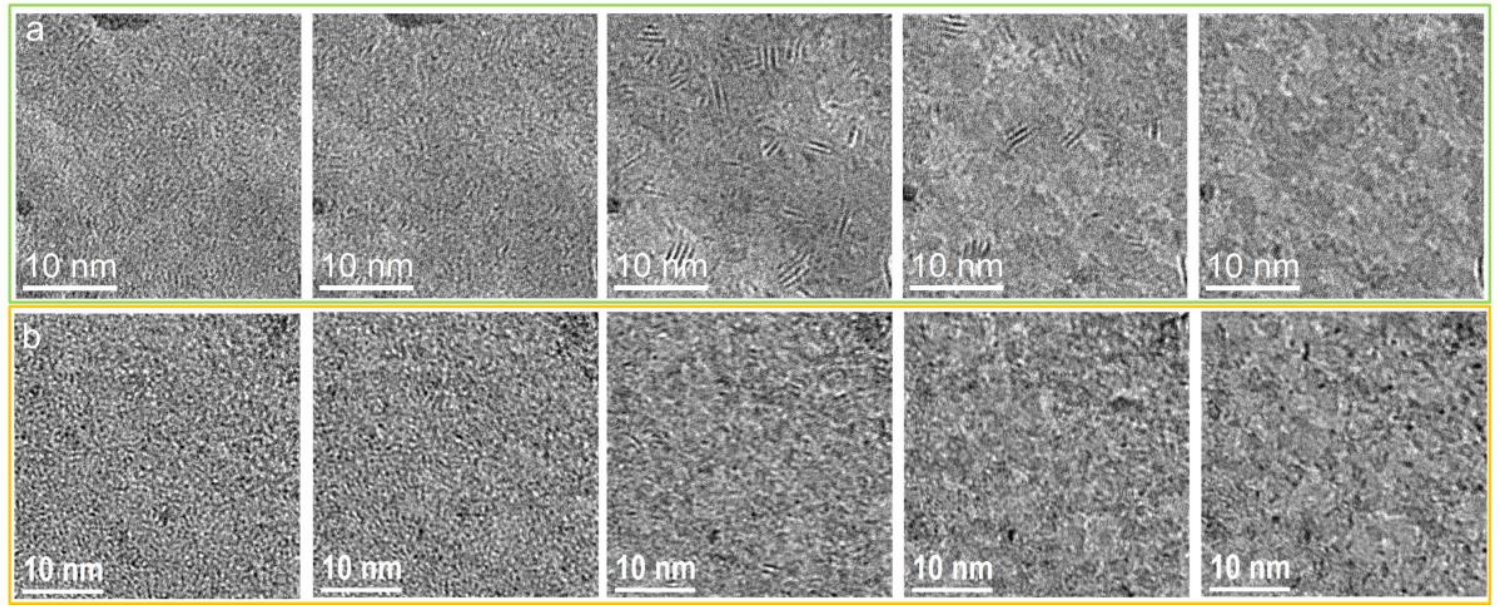

Figure 2. Growth of 2D WSe2 by laser irradiation within the TEM evolved with increasing laser energy on (a) graphene and (b) CVD grown monolayer MoSe2

\section{References}

1. Wu, Y.; Liu, C.; Moore, T. M.et al. Microscopy and Microanalysis 2018, 24 (6), 647-656.

2. Liu, C.; Wu, Y.; Hu, Z. et al. ACS Photonics 2019, 6 (10), 2499-2508.

3. Fei, L.; Lei, S.; Zhang, W.-B. et al. Nature communications 2016, 7, 12206. 